\title{
Safety and efficacy of endoscopic ultrasound-guided gastro- enterostomy using double balloon occlusion methods: a clinical retrospective study in 36 patients with malignant gastric outlet obstruction
}

\section{다)(i) $(5)$}

\author{
Authors \\ Guifang Xu*, ${ }^{*}$, Yonghua Shen ${ }^{*},{ }^{1}$, Ying Lv ${ }^{1}$, Xiaoliang Zhou ${ }^{1}$, Wen Li ${ }^{1}$, Yi Wang ${ }^{1}$, Shahzeb Hassan², Lei Wang ${ }^{1}$, Xiaoping \\ Zou' ${ }^{1}$
}

Institutions

1 Department of Gastroenterology, Affiliated Drum Tower Hospital of Nanjing University Medical School

2 Northwestern University Feinberg School of Medicine, Chicago 60611, IL, United States

submitted 10.12 .2019

accepted after revision 30.6.2020

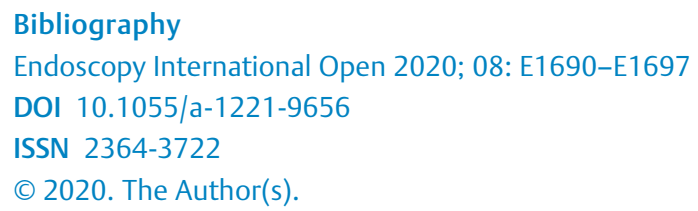
Commons Attribution-NonDerivative-NonCommercial License, permitting copying and reproduction so long as the original work is given appropriate credit. Contents may not be used for commecial purposes, or adapted, remixed, transformed or built upon. (https://creativecommons.org/licenses/by-nc-nd/4.0/)

Corresponding authors

Dr. Xiaoping Zou, The Affiliated Drum Tower Hospital of Nanjing University Medical School, 321 Zhongshan Road, Nanjing, Jiangsu 210008, China

Fax: $+86-25-83105206$

13770773971@163.com

\section{ABSTRACT}

Background and study aims Gastric outlet obstruction (GOO) is common in the late stage of many malignant tumors of the digestive system. Endoscopic ultrasound (EUS)-guided gastroenterostomy (EUS-GE) is commonly used for palliative treatment of malignant GOO. The objec- tive of this study was to investigate the safety, efficacy, and prognosis of EUS-GE in treatment of malignant GOO in Chinese patients.

Patients and methods This was a retrospective, singlecenter study with 36 consecutive patients with malignant GOO who were treated with EUS-GE. The main outcome measures were technical success rate, clinical success rate, incidence of adverse events (AEs), and median survival time.

Results A total of 36 patients with malignant GOO underwent double-balloon-assisted EUS-GE between March 2017 and June 2019 in our hospital. GOO occurred mainly in elderly men (mean age 69.0 years, M:F 0.89). The most common etiology of GOO was pancreatic cancer $(41.7 \%)$. The most common obstruction site was the second part of the duodenum (63.9\%). The technical success rate was 100\% (36/36). The clinical success rate was $94.4 \%$ (34/36). Median time for the total procedure was 52 minutes (range 34 $156 \mathrm{~min}$ ). Median time for determination of puncture site was 20 minutes (range 15-28 min). Median time between puncture and successful delivery of the stent was 38 minutes (range $19-128 \mathrm{~min}$ ). The GOOSS score was 0.2 before EUS-GE. The GOO Scoring System (GOOSS) score was 2.2 at 15 days after the EUS-GE $(P=0.001)$. The GOOSS score was still higher than 2 during a median follow-up period of 89 days. AEs were observed in nine patients $(25.0 \%)$ and 13 total AEs occurred. One patient died as a result of delayed stent migration and bleeding. Mean length of hospital stay was $5.8 \pm 4.7$ days. The median survival period was 103 days. The rate of GOO recurrence was $2.7 \%(1 / 36)$.

Conclusion EUS-GE was associated with increased safety and efficacy for treatment of malignant GOO in Chinese Mainland.

\section{Introduction}

Gastric outlet obstruction (GOO) is very common in the late stage of many malignant tumors of the digestive system, such

\footnotetext{
* These authors contributed equally to the work.
}

as gastric, pancreatic, periampullary, and duodenal cancers [1]. GOO has a serious impact on patient quality of life because it is often associated with symptoms such as nausea, vomiting, and loss of appetite [2,3]. 
Palliative therapies for malignant GOO include gastroenterostomy, endoscopic placement of luminal stent, and total parenteral nutrition (TPN). TPN not only reduces patient quality of life, it also increases medical expenses. Endoscopic placement of an enteric self-expanding metal stent (SEMS) across the malignant stricture is an alternative treatment option. With this procedure, patients can resume oral intake of food, but it may be complicated by recurrent obstruction [1-3]. Patients can also return to oral intake after the gastroenterostomy procedure. Gastroenterostomy includes surgical gastroenterostomy and endoscopic ultrasound (EUS)-guided gastroenterostomy (EUS-GE). Surgical gastroenterostomy has become the standard palliative therapy for malignant gastric outlet obstruction caused by carcinoma of the stomach, duodenum or pancreas. However, many patients tend to be poor surgical candidates due to malnutrition [2]. The surgical approach is invasive and can be associated with significant surgical mortality [4-8]. With the advent of EUS skills and luminal stents, EUS-GE has been accepted as a palliative treatment for malignant GOO because it is a less invasive and long-lasting luminal patency method [3]. Since Fritscher [9] introduced the EUS-GE procedure in pigs, many studies have researched it as a treatment option malignant GOO [10-12]. According to these studies, EUSGE results in recovery of oral intake without risk of tumor ingrowth, while also avoiding the potential morbidity of surgery.

EUS-GE has not been commonly used in Mainland China. However, GOO is very common in China because of the high incidence of gastric and pancreatic cancer. The aim of this study was to demonstrate the feasibility, safety, and short- and longterm prognosis of EUS-GE.

\section{Patients and methods}

\section{Patients}

This was a single-center study. We retrospectively reviewed electronic endoscopy records from March 2017 to June 2019 and 36 consecutive patients with malignant GOO who underwent EUS-GE at the Affiliated Drum Tower Hospital of Nanjing University Medical School. Malignant GOO was diagnosed based on clinical symptoms, laboratory examinations, and imaging (including transabdominal ultrasound, computed tomography scan of the abdomen, magnetic resonance cholangiopancreatography, and EUS). Inclusion criteria consisted of primary or metastatic malignancies that resulted in symptomatic GOO rated with the GOO Scoring System (GOOSS). The GOOSS is based on a score of 0 to 4 ( 0 (no oral intake), 1 (liquid only), 2 (half fluid only), 3 (almost normal diet) and 4 (normal diet)). Contraindications included presence of severe ascites, benign GOO, gastric body tumor, or complete GOO thath could not be passed by the guidewire. We reviewed each patient's endoscopic and medical records and collected information on demographics, etiology of GOO, symptoms, signs, time of tumor diagnosis, time of GOO occurrence, GOOSS scores before treatment, technical success rate, clinical success rate, procedurerelated adverse events (AEs) and post-procedural length of hospital stay. As described by Suzanne M et al. [13] previously, food intake was measured daily during the first 30 days after treat- ment and then weekly by using patient diaries. GOOSS score was used to evaluate food intake. Clinical success was defined as patient ability to tolerate oral intake without vomiting. Health-related quality of life (HRQoL) was assessed with a standardized QoL, the EuroQol-5D (EQ-5D).

The endpoint of observation was June 20, 2019. Follow-up continued from the procedure to the death of patients or to the end of the study. Patients received telephone follow-up by specially trained research nurses at 14 days, 1 month, and then monthly following the procedure, to inquire about complications and record symptoms, signs, recurrent obstruction, GOOSS scores after treatment, and survival time. Depending on their conditions, patients were referred for outpatient or inpatient treatment as indicated. Data collected during follow-up included laboratory tests for liver and kidney functions and imaging results. Follow-up data were collected prospectively. Informed consent was obtained from all patients prior to the endoscopic procedure. The study protocol was approved by the Ethics Committee of the Nanjing Drum Tower Hospital.

\section{Definitions}

AEs were defined as clinically important complications that were considered to be definitely or probably related to EUS$G E$. Delayed bleeding was defined as hemorrhage that occurred 24 hours after the procedure. The metal stent stripping the guidewire coating was defined as the metal stent inserting under the guidewire and stripping the guidewire, causing the insulating layer of the guidewire to fall off and influence stent placement.

\section{Procedure}

Patients were not given antibiotics, but were instructed to fast for 2 days before their scheduled EUS-GE. The procedure was performed by two experienced endoscopists (Xiaoping Zou and Lei Wang), as previously described by Khashab et al. and Itoi et al. [14,15] ( $\mathbf{F i g . 1 ) . ~ B o t h ~ e x p e r t s ~ h a d ~ i n d e p e n d e n t l y ~}$ performed EUS-guided interventions in more than 300 cases. EUS-GE was performed using a double balloon-assisted technique to build a gastroenterostomy bypass. First, a $100-\mathrm{cm}$ enteroscopy overtube was placed outside the upper gastrointestinal endoscope. The endoscope was advanced to the stenosis of the duodenum. A 0.025-inch guidewire (Olympus Medical Systems, Tokyo, Japan) was then maximally advanced. The endoscope was removed, leaving the guidewire and the enteroscopy overtube in place. The guidewire was then inserted across the obstruction to the jejunum. A double balloon with triple cavities (Tokyo Medical University type; Create Medic, Yokohama, China) was inserted over the guidewire to the distal duodenum or the deep small bowel under fluoroscopic assistance. The enteroscopy overtube was subsequently removed. The double balloons were filled with saline and contrast to let the small intestine open. Saline and contrast with methylene blue were injected into the space between the two balloons. The fluid-filled distal duodenum or the proximal jejunum adjacent to the gastric body was identified by the curved linear array echo endoscope (GF-UCT260, Olympus, Tokyo, Japan). When the deep distended jejunum was identified, a 19-gauge 

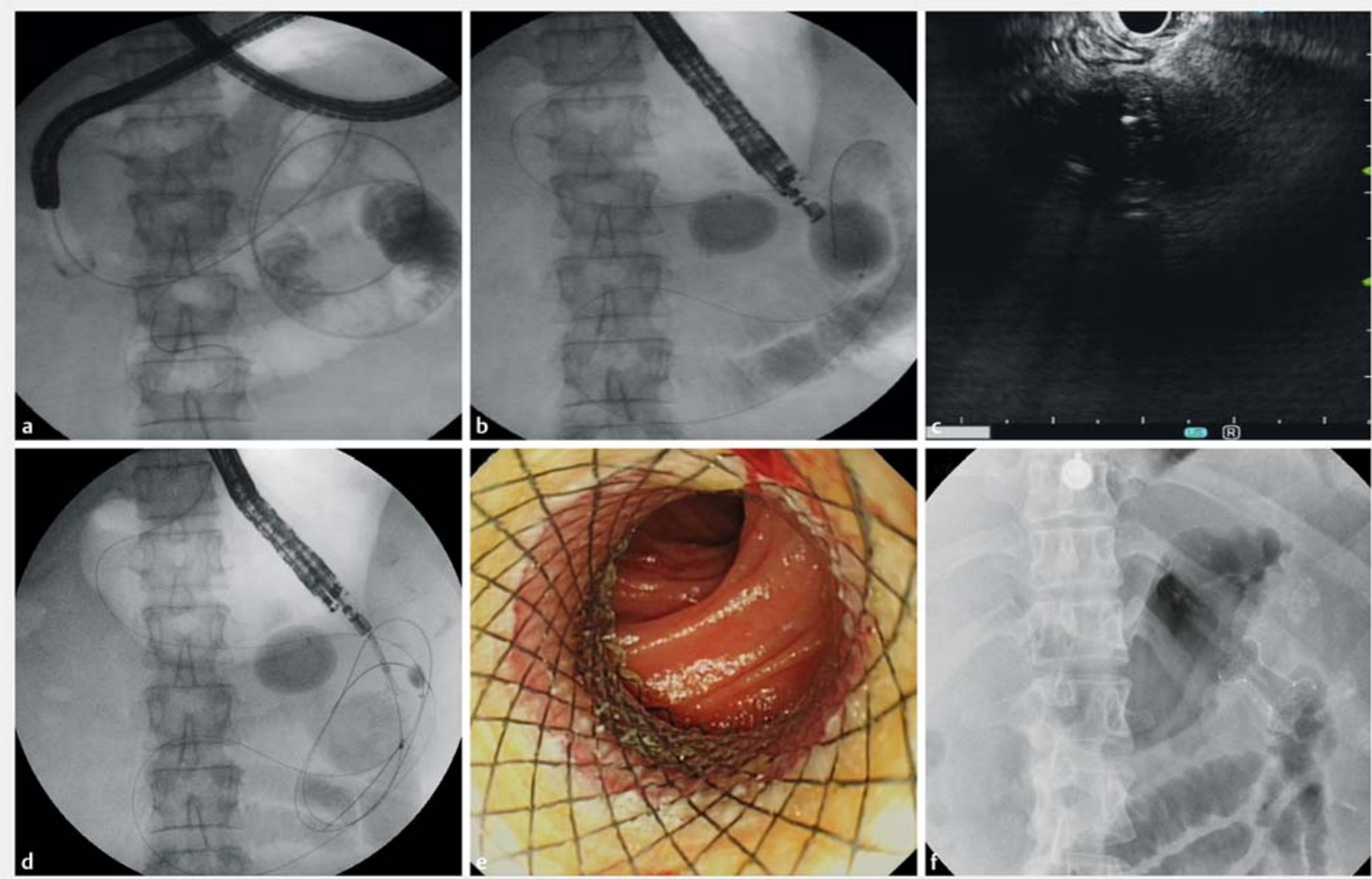

- Fig. 1 Endoscopic ultrasound (EUS)-guided gastroenterostomy for malignant gastric outlet obstruction. a Fluoroscopic view of guidewire inserting across the obstruction. b Fluoroscopic view of a double balloon with triple cavities inserting into the jejunum and filling with saline and contrast. c The distal anchor flange of the stent was deployed under the EUS view. $\mathbf{d}$ The distal anchor flange of the stent was deployed under the fluoroscopic view. e Proximal flange was deployed under endoscopic imaging. f Fluoroscopic view of the completely deployed stent.

fine aspiration needle (EchoTip Ultra; Cook Medical) was used to puncture the jejunum under EUS guidance. The blue fluid was aspirated to demonstrate that the needle was in the jejunum. A 0.035-inch guidewire was advanced through the 19-gauge needle. A lumen-apposing self-expanding metal stent with electrocautery (Micro-Tech (Nanjing) Co., Ltd.) was inserted through the stomach wall into the jejunum wall. The distal anchor flange of the stent was deployed under EUS and fluoroscopic guidance. Then the proximal anchor flange was deployed under endoscopic guidance. The stent lumen was dilated with a $10-\mathrm{mm}$ dilating balloon.

\section{Statistical analysis}

Statistical analysis was performed with SPSS statistical program (SPSS 20.0, Chicago, Illinois, United States). Clinical, endoscopic, and histopathologic characteristics of GOO and patient outcomes were analyzed. Continuous data were expressed as mean \pm standard deviation (SD). Statistical analysis was performed using the student's $t$-test and the Mann Whitney U for normally distributed and non-normally continuous variables, respectively. For categorical variables, data weres also presented as value (\%). The $x^{2}$ test or Fisher's exact test was used to evaluate differences in proportions. $P<0.05$ was considered statistically significant.

\section{Results}

\section{Demographics}

As shown in $>$ Table 1, the mean age of GOO patients was 69.0 years (range $45-88$ years). The male-to-female ratio was 0.89 . The mean time of tumor history was 4 months (range 1-36 months). The mean time of GOO occurrence before EUS-GE was 2 months (range $0.25-3$ months). Reported symptoms at the time for EUS-GE included abdominal pain in 24 (66.7\%), nausea in 31 (86.1\%), vomiting in 31 (86.1\%), jaundice in nine (25.0\%), fever in four (11.1\%) and weight loss in 33 (91.7\%). The most common sign was abdominal tenderness in 16 (44.4 $\%)$. Seven patients (19.4\%) had a history of surgery. The most common cause of $\mathrm{GOO}$ in this study was pancreatic cancer (15/36, 41.7\%), followed by cholangiocarcinoma (8/36, 22.2 $\%)$, duodenal cancer $(5 / 36,13.9 \%)$, metastatic cancer $(4 / 36$, $11.1 \%)$, and gastric cancer $(4 / 36,11.1 \%)$. The average body mass index was 17.9 (range 17-27). The most common obstruction site was the second part of the duodenum $(23 / 36$, $63.9 \%)$, followed by the pylorus $(7 / 36,19.4 \%)$. For 10 patients, 
- Table 1 Baseline characteristics of 36 patients with malignant GOO undergoing EUS-GE.

\begin{tabular}{|c|c|}
\hline Age $(y)$, mean $\pm S D$ & $69.0 \pm 12.8$ \\
\hline \multicolumn{2}{|l|}{ Sex, n (\%) } \\
\hline - Male & $17(47.2)$ \\
\hline Time of tumor history (months), median & 4.0(range 1 36) \\
\hline $\begin{array}{l}\text { Time of GOO before treatment (months), } \\
\text { median }\end{array}$ & $2.0($ range $0.25 \sim 3$ ) \\
\hline GOOSS score, median & $0.2(0 \sim 1)$ \\
\hline \multicolumn{2}{|l|}{ Symptoms, n(\%) } \\
\hline - Abdominal pain & $24(66.7 \%)$ \\
\hline - Nausea & $31(86.1 \%)$ \\
\hline - Vomiting & $31(86.1 \%)$ \\
\hline - Weight loss & $33(91.7 \%)$ \\
\hline - Fever & $4(11.1 \%)$ \\
\hline - Jaundice & $9(25.0 \%)$ \\
\hline \multicolumn{2}{|l|}{ Signs, n (\%) } \\
\hline - Abdominal tenderness & $16(44.4 \%)$ \\
\hline - Anaemic appearance & $12(33.3 \%)$ \\
\hline \multicolumn{2}{|c|}{ Etiology of malignant gastric outlet obstruction, n (\%) } \\
\hline - Gastric cancer & $4(11.1 \%)$ \\
\hline - Pancreatic cancer & $15(41.7 \%)$ \\
\hline - Cholangiocarcinoma & $8(22.20 \%)$ \\
\hline - Duodenal cancer & $5(13.9 \%)$ \\
\hline - Metastatic cancer & $4(11.1 \%)$ \\
\hline \multicolumn{2}{|l|}{ Site of the obstruction, $\mathrm{n}(\%)$} \\
\hline - Pyloric & $7(19.4 \%)$ \\
\hline - First part of the duodenum & $4(11.1 \%)$ \\
\hline - Second part of the duodenum & $23(63.9)$ \\
\hline - Third part of the duodenum & $2(5.6 \%)$ \\
\hline \multicolumn{2}{|l|}{ concomitance's disease } \\
\hline - Hypertension & $14(38.9 \%)$ \\
\hline - Diabetes & $12(33.3 \%)$ \\
\hline $\begin{array}{l}\text { GOO, gastric outlet obstruction; EUS-GE, endo } \\
\text { gastroenterostomy }\end{array}$ & ultrasound-guided \\
\hline
\end{tabular}

enteral stenting (ES) was performed one to three times and the mean time without obstruction was 78 days.

\section{Outcomes of the EUS-GE}

- Table 2 shows outcomes of 36 patients who underwent EUSGE. All patients $(36 / 36,100 \%)$ underwent double balloon-assisted EUS-GE. The site of puncture was the posterior wall of gastric body in 36 patients (36/36, 100\%). The mean number of punctures was 1.3 (range $1 \sim 3$ times). Nine patients received
- Table 2 Outcomes for 36 patients that underwent EUS-GE.

\begin{tabular}{|c|c|}
\hline \multicolumn{2}{|l|}{ Site of puncture, n (\%) } \\
\hline - Posterior wall of gastric body & $36(100 \%)$ \\
\hline Number of punctures, mean \pm SD & $1.3 \pm 0.7$ \\
\hline Total procedure time(minutes), median \pm SD & $52.0(34-156)$ \\
\hline $\begin{array}{l}\text { Time for determination of puncture site } \\
\text { (minutes), median } \pm S D\end{array}$ & $20(15-28)$ \\
\hline $\begin{array}{l}\text { Time between puncture to stent delivery } \\
\text { (minutes), median } \pm \text { SD }\end{array}$ & $38(19-128)$ \\
\hline Technical success, n (\%) & $36(100 \%)$ \\
\hline Clinical success, n (\%) & $34(94.4 \%)$ \\
\hline \multicolumn{2}{|l|}{ Adverse events during procedure, $\mathrm{n}(\%)$} \\
\hline - Bleeding & $2(5.6 \%)$ \\
\hline - Stent misdeployment & $1(2.8 \%)$ \\
\hline - The mental stent cutting the guidewire & $3(8.3 \%)$ \\
\hline \multicolumn{2}{|l|}{ Adverse events after procedure, $\mathrm{n}(\%)$} \\
\hline - Stent migration after procedure & $2(5.6 \%)$ \\
\hline - Peritonitis & $3(8.3 \%)$ \\
\hline - Delayed bleeding & $2(5.6 \%)$ \\
\hline
\end{tabular}

more than one puncture and the most likely cause was most likely low position of obstruction. Median total procedure time was 52 minutes (range 34 156 min). Median time for determination of puncture site was 20 minutes (range 15 28 min). Median time from puncture to successful delivery of the stent was 38 minutes (range 19 128 min). Significantly less time was spent on determination of puncture site than for stent placement $(P=0.001)$. The technical success rate was $100 \%(36 / 36)$. The clinical success rate was $94.4 \%(34 / 36)$.

AEs were seen in nine patients $(9 / 36,25.0 \%)$ and 13 total AEs occurred. During the procedure, three patients $(3 / 36,8.3 \%)$ had the metal stent stripped of guidewire coating, which affected the ability of the cutting current to penetrate the metal stent through the gastrointestinal wall. Replacement of guidewire or additional puncture became a remedy. The total procedure time was 109 minutes per patient. One patient had stent misdeployment during the procedure, and the stent was pulled out and a new stent was deployed successfully. After the procedure, stent migration was seen in two patients (2/36, 5.6\%), including one who had the metal stent stripped of guidewire coating ( $\triangleright$ Fig. 2 ) during the procedure, and the new bare metal stent was inserted into the covered metal stent. After the new stent insertion, one patient had an AE and bled severely. Endoscopy showed that blood oozed from the puncture site and the patient died from failure of endoscopic hemostasis and conservative treatment. For the other patient, when the new baremetal stent was unable to control the bleeding, the two stents, including the bare and the covered one, were pulled out. Then 


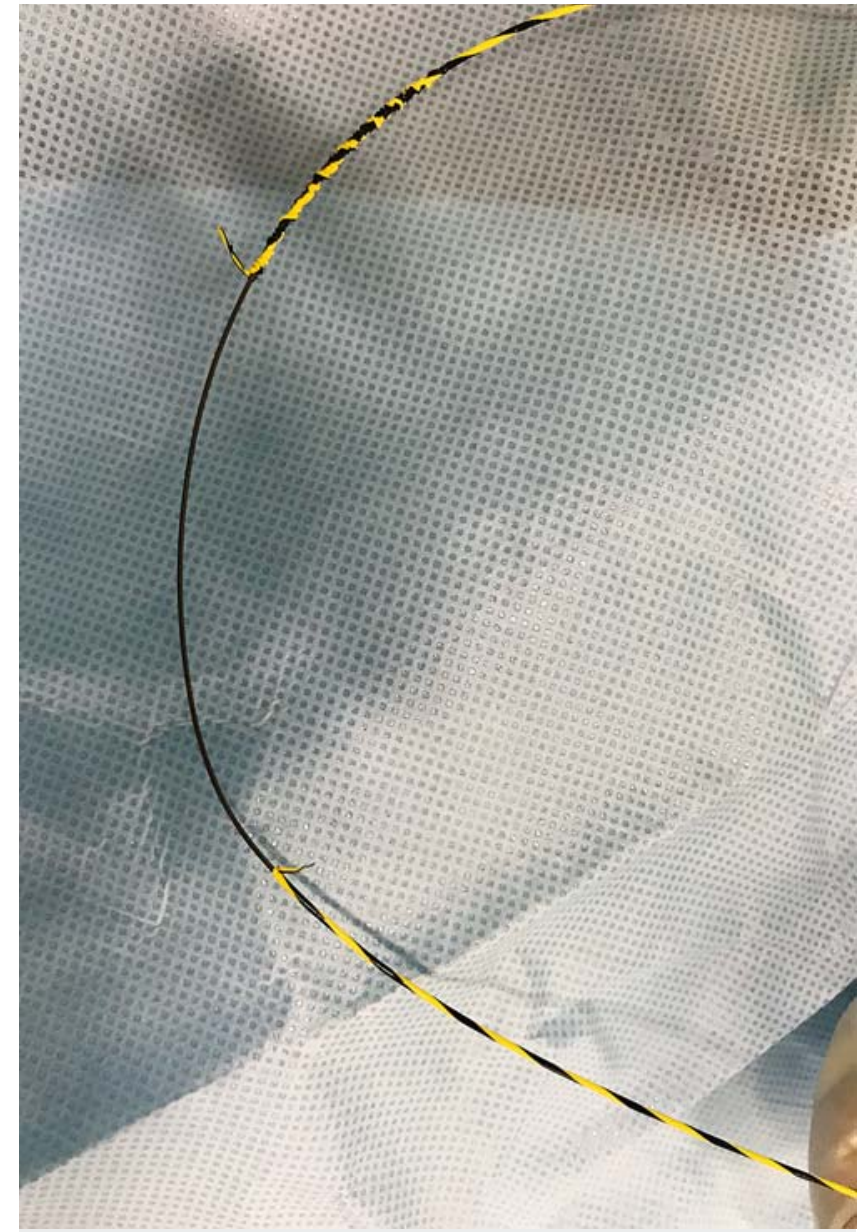

Fig. 2 The mental stent stripping the guidewire coating. the guidewire was inserted through the puncture site into the jejunum, and the new covered metal stent was placed, leading to resolution of the bleeding. In seven patients, postoperative pain occurred in seven patients, which required readmittance within 1 to 4 days after conservative treatment. No peritonitis occurred. No bleeding occurred during the procedure.

\section{Quality of life and prognosis}

- Fig. 3 shows food intake and EQ-5D scores before EUS-GE and after EUS-GE. Only seven patients ate a little fluid food and the other 29 patients were unable to eat any food. The GOOSS score was 0.2 before EUS-GE. Food intake improved rapidly after EUS-GE. After stent placement, ability to eat was restored in a median of 2 days. The GOOSS score was 2.2 at 15 days after EUS-GE, which was higher than before EUS-GE $(P=0.001)$. During the follow-up period, the GOOSS was still higher than 2 at $30,60,90,120,150$, and 180 days. EQ-5D scores showed a slight increase after EUS-GE within 60 days, and then they gradually went down.

Post-procedure length of hospital stay was $5.8 \pm 4.7$ days. Median follow-up time was 89 days. The survival curve for patients undergoing EUS-GE is shown in > Fig. 4. Median survival time was 103 days. The rate of GOO recurrence was $2.7 \%$ (1/36, $2.7 \%)$. The endoscopic views showed that the stent was patent and iodine water radiography showed that the iodine water flowed from the stomach to the jejenum. However, symptoms of vomiting occurred again and the patient was not able to eat 56 days after the procedure. Reexamination of computed tomography showed that the tumor had metastasized to the abdominal cavity, resulting in downstream intestinal congestion.

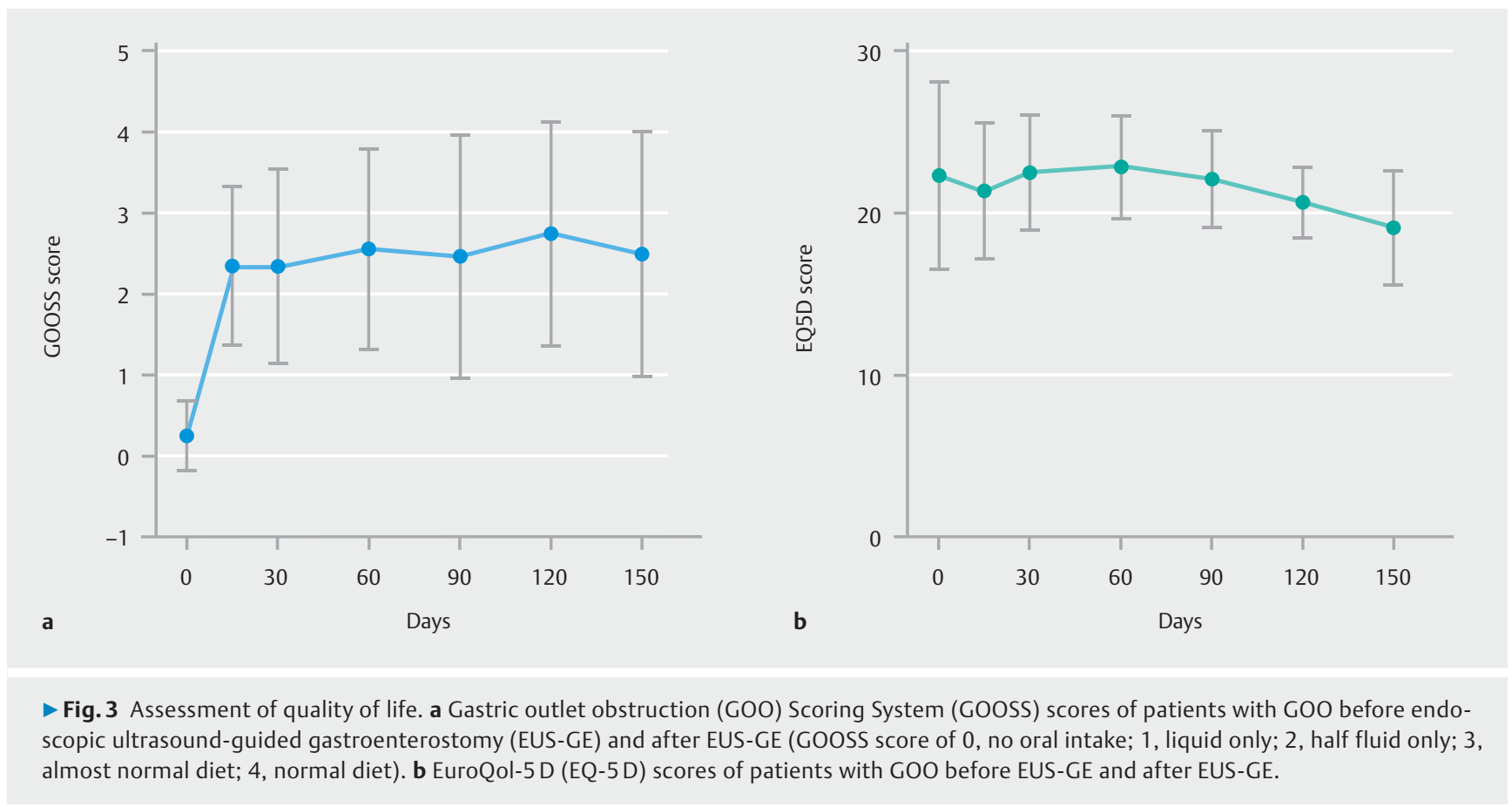




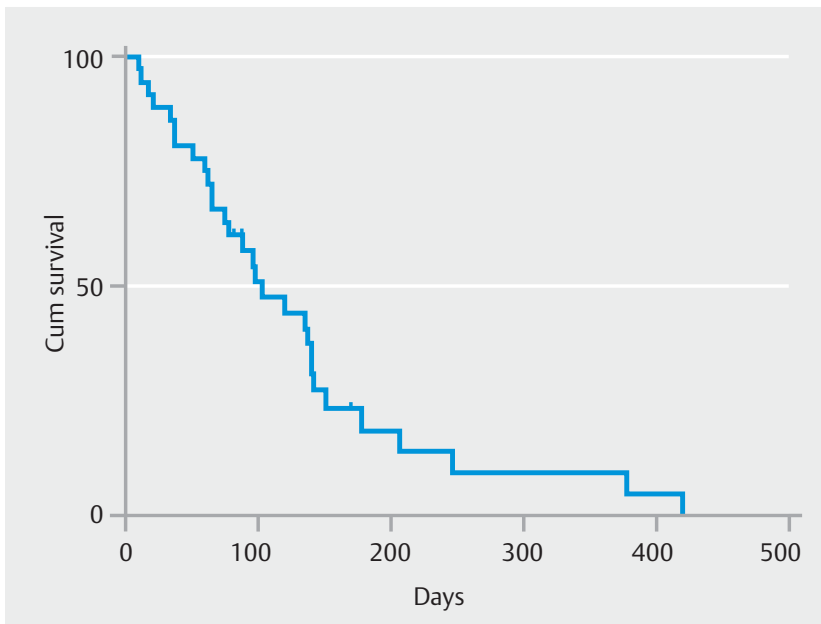

- Fig. 4 Survival curves of patients undergoing endoscopic ultrasound-guided gastroenterostomy.

\section{Discussion}

$\mathrm{GOO}$ is very common in China because of the high incidence of malignant tumors of the the digestive system. Most of these patients have advanced, unresectable tumors and thus, symptom palliation and improvement in quality of life are the usual goals of any intervention [16]. In 2002, Fritscher-Ravens et al. first reported on EUS-guided gastrojejunostomy in pigs. In recent years, EUS-GE has been used as a palliative treatment for malignant GOO. Several clinical and animal studies have shown that the technical success rate with it is $86.7 \%$ to $100 \%$. In clinical studies, success rates range from $83.3 \%$ to $100 \%[9-12,15$, $17-20]$.

This trial used palliative EUS-GE to treat late malignant GOO. In this study, 36 patients with malignant GOO were included and the most common etiology for GOO was pancreatic cancer. All patients underwent balloon-assisted EUS-GE. We selected the metal covered stent with electrocautery delivery system, which supports electrocautery dilation and stent deployment without the need for primary dilation. The site of puncture was at the posterior wall of gastric body in 36 patients (100\%). The technical success rate was $100 \%$, and the clinical success rate was $94.4 \%$. Food intake improved after EUS-GE. GOOSS scores increased from 0.2 to 2.2. Most patients could eat soft food without vomiting. If the stent was patent, the GOOSS score increased to more than 2 at 180 days after the procedure. Overall, our data suggest that EUS-GE is a very effective treatment for symptomatic GOO in China. A long-term cohort study has shown that the rate of reintervention is low with the EUS-GE approach [21].

EUS-GE has some complications, including bleeding, stent misdeployment, and recurrence of obstruction [20,22]. Chen $\mathrm{YI}$ et al. reported a rate of severe AEs of $10 \%$ in the EUS-GE group. However, overall rates of AEs were not significantly different between the EUS-GE group and the endoscopic ES group [20]. Previous studies showed that stent occlusion was very common in the ES group $[3,23,24]$. Compared to ES place- ment, EUS-GE has a lower rate of stent failure requiring repeat intervention [25]. However, the covered enteral stent has higher rates of stent migration and tumor overgrowth remains a significant problem in ES [26-28]. Mouen A et al. reported three misdeployments of the stent first flange in the peritoneum and two episodes of abdominal pain requiring hospitalization. Stent misdeployment prolonged hospitalization, but conservative treatment with antibiotics was safe [22]. Tyberg A et al. also reported one death in a severely debilitated patient, which was associated with procedure-related peritonitis [10]. Our data show that procedure-associated complications are metal stent stripping the guidewire coating and stent misdeployment. The insulated outer sheath of the guidewire was cut down and covered on the front end of the electrocoagulation part of the metal stent, which affected cutting current penetration into the gastrointestinal wall. In one patient, the metal stent cut the guidewire, prolonging the procedure time. Stent misdeployment occurred in one patient during the procedure. We pulled out the stent and successfully deployed a new stent, and no severe AE occurred. Post-procedure AEs included stent migration in two patients, and the new bare metal stent was inserted into the primary stent. One patient experienced severe bleeding and died due to failure of endoscopic and conservative treatment and patient refusal of surgical treatment. In the other patient, when the second stent was found to be ineffective, the two stents were pulled out quickly. We deployed a new metal-covered stent, which controlled the bleeding. The patient recovered with conservative treatment. In this study, we had only one case of severe bleeding after EUS-GE. Overall, we found that the procedure was safe for most of our patients. However, the reasons for stent migration need to be evaluated carefully before a new bare stent is deployed into the primary stent, to avoid fatal complications.

In this study we used a LA-FSEMS with cautery tip. The distal end of the stent delivery system was inserted through the stomach wall into the jejunal lumen by applying electrocautery. This delivery system does not require balloon catheters or endoscopic removal before stent insertion. This type of stent, with the same function, is manufactured by several different companies [10 - 11]. Our results showed that a metal stent cutting the guidewire could affect electrocoagulation and prolong the procedure time. During the procedure, cutting of the guidewire by the stent is confirmed, the guidewire should be reinserted into the jejunum and the damaged guidewire kept far away from the puncture area. The alternative is to pull out the guidewire and stents and do the puncture again. In our study, two patients had stent migration after the procedure. In the future, research on stent choice to avoid migration should be undertaken, including size and width of biflanges, and apposing properties.

During EUS-GE, it is difficult to puncture the jejunum because the jejunum is highly mobile and can be easily moved away from the stomach. We undertook EUS-guided double-balloon-occluded gastroenterostomy and chose to puncture the small bowel around the ligament of Treitz because that part of the intestine is adjacent to the stomach. In this study, puncture was successfully in 36 patients. A 19-gauge FNA needle was 
used to puncture the jejunum (between the two balloons) rather than the balloon with filled saline and contrast because the balloon could have affected the electrocoagulation. It was not appropriate to perform EUS-GE when the tumor had expanded to the jejunum around the ligament [29].

Mean length of hospital stay for EUS-GE in a comparative trial of endoscopic it versus surgical gastrojejunostomy was 11.6 days. However, mean length of hospital stay for EUS-GE was 12 days [22]. The first US clinical study of EUS-GE demonstrated that mean length of hospital stay was 2.2 days [11]. Comparatively, our data showed that mean length of hospital stay was 5.8 days. Although the other study demonstrated that there was no symptom recurrence during a mean follow-up period of 150 days [11], previous studies have reported symptom recurrence during the follow-up period because of stent obstruction with food, which was successfully treated with endoscopic extraction [22]. One patient in our study had recurrence of symptoms. Neither stent migration nor obstruction could be confirmed with endoscopy or iodine water. However, the patient had symptoms of vomiting and nausea 56 days after the procedure. Median survival time after EUS-GE was 103 days in two previous studies $[20,22]$. It was the same in our study, which showed that EUS-GE is valuable for many patients with advanced malignant GOO. Overall, EUS-GE has many advantages, such as minimal hospital stay after the procedure, lower rate of symptom recurrence, and improved overall survival.

There were several limitations to our study. First, it was single-center and retrospective. Second, surgical gastroenterostomy and endoscopic enteral stenting were not compared. Third, only one type of stent was used and there was one fatal $A E$. In the future, potential for AEs during and after the procedure should be controlled. Multicenter, prospective, and comparative studies should be performed of endoscopic versus surgical gastroenterostomy and endoscopic enteral stenting for malignant GOO.

\section{Conclusion}

In conclusion, our results indicated that EUS-GE was safe and effective. EUS-GE has many advantages for patients with advanced malignant $\mathrm{GOO}$, such as its minimally invasive nature, requirement for only a short postoperative stay, lower rate of symptom recurrence, and improved overall survival rates. However, the procedure is challenging procedure and should only be performed by experienced physicians. Moreover, appropriate patient selection for EUS-GE is important. Overall, EUS-GE is a promising treatment option for malignant $\mathrm{GOO}$ and proper care should be used when considering patients for this procedure.

\section{Competing interests}

The authors declare that they have no conflict of interest.

\section{References}

[1] Espinel J, Vivas S, Munoz F et al. Palliative treatment of malignant obstruction of gastric outlet using an endoscopically placed enteral Wallstent. Dig Dis Sci 2001; 46: 2322-2324

[2] Adler DG, Baron TH. Endoscopic palliation of malignant gastric outlet obstruction using self-expanding metal stents: experience in $36 \mathrm{pa}-$ tients. Am J Gastroenterol 2002; 97: 72-78

[3] Oh SY, Edwards A, Mandelson M et al. Survival and clinical outcome after endoscopic duodenal stent placement for malignant gastric outlet obstruction: comparison of pancreatic cancer and nonpancreatic cancer. Gastrointest Endosc 2015; 82: 460-468 e462

[4] Del Piano M, Ballare M, Montino F et al. Endoscopy or surgery for malignant Gl outlet obstruction? Gastrointest Endosc 2005; 61: 421-426

[5] Johnsson E, Thune A, Liedman B. Palliation of malignant gastroduodenal obstruction with open surgical bypass or endoscopic stenting: clinical outcome and health economic evaluation. World J Surg 2004; 28: 812-817

[6] Maetani I, Tada T, Ukita T et al. Comparison of duodenal stent placement with surgical gastrojejunostomy for palliation in patients with duodenal obstructions caused by pancreaticobiliary malignancies. Endoscopy 2004; 36: 73-78

[7] Mittal A, Windsor J, Woodfield J et al. Matched study of three methods for palliation of malignant pyloroduodenal obstruction. $\mathrm{Br}$ J Surg 2004; 91: 205-209

[8] Watanapa P, Williamson RC. Surgical palliation for pancreatic cancer: developments during the past two decades. $\mathrm{Br}$ J Surg 1992; 79: 8-20

[9] Fritscher-Ravens A, Mosse CA, Mukherjee D et al. Transluminal endosurgery: single lumen access anastomotic device for flexible endoscopy. Gastrointest Endosc 2003; 58: 585-591

[10] Tyberg A, Perez-Miranda M, Sanchez-Ocana R et al. Endoscopic ultrasound-guided gastrojejunostomy with a lumen-apposing metal stent: a multicenter, international experience. Endosc Int Open 2016; 4: E276-281

[11] Khashab MA, Kumbhari V, Grimm IS et al. EUS-guided gastroenterostomy: the first U.S. clinical experience (with video). Gastrointest Endosc 2015; 82: 932-938

[12] Binmoeller KF, Shah JN. Endoscopic ultrasound-guided gastroenterostomy using novel tools designed for transluminal therapy: a porcine study. Endoscopy 2012; 44: 499-503

[13] Jeurnink SM, Steyerberg EW, van Hooft JE et al. Surgical gastrojejunostomy or endoscopic stent placement for the palliation of malignant gastric outlet obstruction (SUSTENT study): a multicenter randomized trial. Gastrointest Endosc 2010; 71: 490-499

[14] Khashab MA, Baron TH, Binmoeller KF et al. EUS-guided gastroenterostomy: a new promising technique in evolution. Gastrointest Endosc 2015; 81: 1234-1236

[15] Itoi T, Ishii K, Ikeuchi $\mathrm{N}$ et al. Prospective evaluation of endoscopic ultrasonography-guided double-balloon-occluded gastrojejunostomy bypass (EPASS) for malignant gastric outlet obstruction. Gut 2016; 65: 193-195

[16] van Heek NT, van Geenen RC, Busch OR et al. Palliative treatment in "peri"-pancreatic carcinoma: stenting or surgical therapy? Acta Gastroenterol Belg 2002; 65: 171-175

[17] Fritscher-Ravens A, Mosse CA, Mills TN et al. A through-the-scope device for suturing and tissue approximation under EUS control. Gastrointest Endosc 2002; 56: 737-742

[18] Binmoeller KB, Shah J. Endoscopic ultrasound-guided gastroenterostomy using novel tools designed for transluminal therapy: a porcine study. Endoscopy 2012; 44: 499-503 
[19] Itoi T, Itokawa F, Uraoka T et al. Novel EUS-guided gastrojejunostomy technique using a new double-balloon enteric tube and lumen-apposing metal stent (with videos). Gastrointest Endosc 2013; 78: 934939

[20] Chen YI, Itoi T, Baron TH et al. Erratum to: EUS-guided gastroenterostomy is comparable to enteral stenting with fewer re-interventions in malignant gastric outlet obstruction. Surg Endosc 2017; 31: 3765

[21] Kerdsirichairat T, Irani S, Yang J et al. Durability and long-term outcomes of direct eus-guided gastroenterostomy using lumen-apposing metal stents for gastric outlet obstruction. Endosc Int Open 2019; 7: E144-E150

[22] Khashab MA, Bukhari M, Baron TH et al. International multicenter comparative trial of endoscopic ultrasonography-guided gastroenterostomy versus surgical gastrojejunostomy for the treatment of malignant gastric outlet obstruction. Endosc Int Open 2017; 5: E275E281

[23] Dormann A, Meisner S, Verin N et al. Self-expanding metal stents for gastroduodenal malignancies: systematic review of their clinical effectiveness. Endoscopy 2004; 36: 543-550
[24] Oh D, Lee SS, Song TJ et al. Efficacy and safety of a partially covered duodenal stent for malignant gastroduodenal obstruction: a pilot study. Gastrointest Endosc 2015; 82: 32-36.e1

[25] Ge PS, Young JY, Dong W et al. EUS-guided gastroenterostomy versus enteral stent placement for palliation of malignant gastric outlet obstruction. Surg Endosc 2019; 33: 3404-3411

[26] Didden P, Spaander MC, de Ridder R et al. Efficacy and safety of a partially covered stent in malignant gastric outlet obstruction: a prospective Western series. Gastrointest Endosc 2013; 77: 664-668

[27] Kim CG, Choi IJ, Lee JY et al. Covered versus uncovered self-expandable metallic stents for palliation of malignant pyloric obstruction in gastric cancer patients: a randomized, prospective study. Gastrointest Endosc 2010; 72: 25-32

[28] Kim ID, Kang DH, Choi CW et al. Prevention of covered enteral stent migration in patients with malignant gastric outlet obstruction: a pilot study of anchoring with endoscopic clips. Scand J Gastroenterol 2010; 45: 100-105

[29] Itoi T, Ishii K, Tanaka R et al. Current status and perspective of endoscopic ultrasonography-guided gastrojejunostomy: endoscopic ultrasonography-guided double-balloon-occluded gastrojejunostomy (with videos). J Hepatobiliary Pancreat Sci 2015; 22: 3-11 